\title{
Cryptococcal Meningitis amomg HIV-Infected and HIV-Uninfected Patients in Jos, North Central Nigeria
}

\author{
Okolo M O, ${ }^{1}$ Onyedibe K I, ${ }^{1}$ Dabe $F^{3}$, Obishakin E $F^{4}$, Envuladu E $A^{2}$, Egah D Z ${ }^{1}$, Hawkins $C^{5}$ \\ ${ }^{I}$ Department of Medical Microbiology and ${ }^{2}$ Department of Community Medicine, College of Health Science, University of Jos, \\ Nigeria. \\ ${ }^{3}$ Department of Family Medicine, Plateau State Specialist Hospital, Jos, Nigeria \\ ${ }^{4}$ Biotechnology, National Veterinary Research Institute, Vom, Plateau state, Nigeria. \\ ${ }^{5}$ Department of Medicine, Northwestern University Feinberg School of Medicine, Chicago, IL, USA
}

*Correspondence: Okolo Mark Ojogba

Email: okolomark@gmail.com,Phone: +234(0)8027022223

\author{
Article information \\ Date Submitted: $\quad 6 / 4 / 2021$ \\ Date Accepted: $\quad$ 17/6/2021 \\ Date Published: $\quad$ 8/7/2021
}

\begin{abstract}
Cryptococcal meningitis is a global opportunistic infection caused by several serovars of Cryptococcus neoformans and Cryptococcus gattii with significant morbidity and mortality particularly in immunocompromised patients. Management principles involve early and specific diagnosis followed by appropriate antifungal therapy. The study was conducted among in-patients with clinical features of meningitis admitted into tertiary healthcare facilities in Jos, north central Nigeria from November 2017 to December 2018. Cerebrospinal fluid and blood samples were collected from the patients for culture and sensitivity, serology and genomic studies. Univariate and bivariate analysis was carried out using STATA (version 14IC). The serologic prevalence of cryptococcal meningitis among patients with clinical features of meningitis was $16.8 \%$. The prevalence among HIV-infected and HIV-uninfected patients were $13.4 \%$ and $18.8 \%$ respectively. The antifungal susceptibility of the Cryptococcus neoformans isolated from sample cultures at MIC90ug/ml to Amphotericin B, Fluconazole and Itraconazole were all within the reference range. Gene accession numbers of cryptococcal isolates deposited in the gene bank include MK886574.1, MK886568.1, MK886570.1, MK886563.1, MK886560.1, MK886573.1, MK886562.1, MK886558.1. Cryptococcus neoformans are a major cause of meningitis in both HIV-infected and HIV-uninfected patients. This study highlights the fact that routine surveillance should be put in place for culture, antifungal susceptibility tests and genomic studies to ascertain the antifungal susceptibility pattern and genotypes of cryptococcus in circulation.
\end{abstract}

Keywords: Cryptococcus, meningitis, antifungal agent, HIV-infected, HIV-uninfected

How to cite this article

Okolo M O, Onyedibe K I, Dabe F, Obishakin E F, Envuladu E A, Egah D Z, et al. Cryptococcal Meningitis amomg HIVInfected and HIV-Uninfected Patients in Jos, North Central Nigeria. J Biomed Res Clin Pract:2021;4(2):1-7. DOI: 10.46912/jbrcp. 221 .

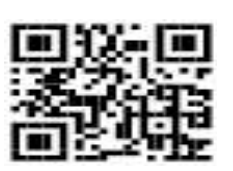

Access to the article

website: http://www.jbrcp.net

do: : https://doi.org/10.46912/jbrcp.221 


\section{INTRODUCTION}

Cing ryptococcal meningitis is a serious systemic fungal infection (mycosis) that mainly affects immunocompromised patients causing high morbidity and mortality. ${ }^{1}$ It is a life-threatening opportunistic infection that is normally observed in the late stages of acquired immunodeficiency syndrome (AIDS). ${ }^{2,3}$ The vast majority of cryptococcal meningitis are caused by two pathogenic species, Cryptococcus neoformans and Cryptococcus gattii which differ in host range, virulence as well as antifungal susceptibility. ${ }^{4-6}$ The disease affects approximately one million persons in the world annually resulting in more than 400,000 deaths within three months following the onset of the disease. ${ }^{1,5,7}$ Cryptococcal meningitis causes high mortality rates in HIV-infected patients (9\%-55\%) and HIV-uninfected patients $(15 \%-44 \%)$, despite optimal care. ${ }^{8,9}$ There is a decline in the incidence of cryptococcal infection in the developed nations of the world due to effective antiretroviral therapy. ${ }^{2,3,10}$

Several classes of antifungal drugs effectively treat cryptococcal infections, but the organisms can develop resistance to these drugs. ${ }^{6,11,12}$ Many in vitro studies have shown that Cryptococcus species are highly susceptible to amphotericin B at a minimum inhibitory concentration (MIC) of $\leq 1 \mu \mathrm{g} / \mathrm{ml}$, susceptible to flucytosine at an MIC of $\leq 4 \mu \mathrm{g} / \mathrm{ml}$, fluconazole at $\leq 8 \mu \mathrm{g} / \mathrm{ml}^{6,11}$ Fewer studies carried out were mainly on serology to determine the prevalence of cryptococcal meningitis. ${ }^{8,13}$ Molecular methods such as polymerase chain reaction (PCR) and sequencing ${ }^{6,11,14}$ will identify the fungus. Hence the diagnosis and treatment of cryptococcal meningitis remain challenging.

This study aims to determine the cryptococcal agents of meningitis in HIV-infected and HIV-uninfected patients in Jos, Nigeria. We also sought to determine the antifungal susceptibility pattern of the Cryptococcus species.

\section{MATERIALS AND METHODS}

This cross-sectional study was conducted among adult patients with clinical features of meningitis admitted into two tertiary health care facilities in Jos. The patients were recruited based on the following criteria; the presence of meningitis, acceptance of lumbar puncture, and consent to participate. The exclusion criteria for enrollment included: patients with sepsis due to neurologic infection or metabolic abnormality, patients with psychosis and peripheral neuropathy, patients with neurosurgical illnesses. All the HIV-infected patients were diagnosed with HIV infection before presenting with features of meningitis and were already on antiretroviral therapy.

\section{Cerebrospinal fluid analysis}

All cerebrospinal fluid samples were collected via lumbar puncture in aseptic conditions into sterile tubes and sent immediately to the laboratory. Each sample was split into two cryovial tubes; one of each pair of samples was used for microscopic examination by Indian ink, latex agglutination and culture and the second for serologic and genomic study.

\section{Serology/Molecular identification}

Cryptococcal antigen enzyme immunoassay (CrAg EIA) was carried out to detect capsular polysaccharide antigen of Cryptococcus species (IMMY; Inc. U.SA).

Total genomic DNA extraction from the Cryptococcus species isolated on the cultured plates was performed using Zymo Quick-DNA kits reagents (Zymo Research Corporation, USA) per the manufacturer's instructions. Polymerase Chain Reaction (PCR) using specific primer sequence for the internal transcribed spacer 1 and 4 (ITS 1 and ITS 4) regions were used. The PCR products were utilized for sequencing with a Big Dye Terminator Reaction Kit v3.1(Applied Biosystems, Inc., Foster City, CA, USA) according to the manufacturer's instructions. After the purification and denaturation of the products, the samples were run on an automated ABI 3500XL genetic analyser (Applied Biosystems, Inc., Foster City, 
CA, USA). The sequences obtained were aligned and compared with sequences deposited in a genomic database (GenBank, NCBI, USA). To ensure high reliability of the results obtained using the nucleotide sequence alignment tools, ${ }^{15,16}$ an e-value of less than $10^{-5}$ and a maximum identity of equal to or higher than $98 \%$ were considered for the correct identification of Cryptococcus at the species level and the phylogenetic analysis was carried out using Bio Edit, and MEGA 07.

\section{RESULTS}

During the study period, a total of 184(67 HIV-infected and 117 HIV-uninfected) patients with features of meningitis were recruited and $31(16.8 \%)$ of these had cryptococcal meningitis $\{9 \mathrm{HIV}$-infected $(13.4 \%)$ and 22 HIV-uninfected (18.8\%)\}, and male gender was predominant in both groups as shown in Table 1

Table 1 : Demographic and laboratory characteristics of HIV-infected and HIV -uninfected patients with cryptococcal meningitis in Jos, North-Central Nigeria

\begin{tabular}{|c|c|c|c|}
\hline Parameter & HIV+ & HIV- & $\mathbf{P}$ \\
\hline Age mean no years ${ }^{\$}$ & 30.2 & 49.3 & $<0.001$ \\
\hline Number recruited & 67 & 117 & NA \\
\hline Male/Female & $7 / 2$ & $16 / 6$ & $>0.999$ \\
\hline Positive serology & 9 & 22 & 0.416 \\
\hline CSF culture positive/positive serology & $7 / 9$ & $19 / 22$ & 0.613 \\
\hline CSF CrAg titer $\geq 1: 512^{\$} /$ positive serology & $5 / 9$ & $20 / 22$ & 0.043 \\
\hline CSF Indian Ink positive/positive serology & $7 / 9$ & $13 / 22$ & 0.429 \\
\hline $\mathrm{CD} 4 / \mathrm{mm}^{3}$ Median (range) & $32.5(8-10.9)$ & NA & NA \\
\hline HIV viral load $\log _{10}$ copies/ml & $6.18 \pm 0.62$ & NA & NA \\
\hline
\end{tabular}

$P<0.05 ;$ \$ students t test, CSF: Cerebrospinal fluid, CrAg: Cryptococcal antigen, NA: not applicable

Table 2: Antifungal susceptibility of Cryptococcus neoformans isolated from HIV-infected and HIVuninfected patients with meningitis in Jos, North-Central Nigeria

\begin{tabular}{lllll}
\hline Antifungal agents & MIC range $(\boldsymbol{\mu g} / \mathbf{m l})$ & $\mathbf{M I C}_{\mathbf{5 0}}(\boldsymbol{\mu g} / \mathbf{m l})$ & $\mathbf{M I C}_{\mathbf{9 0}}(\boldsymbol{\mu g} / \mathbf{m l})$ & $\mathbf{G M}(\boldsymbol{\mu g} / \mathbf{m l})$ \\
\hline HIV-uninfected: & & & & \\
$\quad$ Amphotericin B & $0.0625-1$ & 0.5 & 1 & 0.5163 \\
Fluconazole & $0.125-3$ & 1 & 4 & 1.1578 \\
Itraconazole & $0.0312-0.5$ & 0.125 & 0.25 & 0.1185 \\
HIV-Infected: & & & & \\
Amphotericin B & $0.125-1$ & 0.5 & 1 & 0.3410 \\
$\quad$ Fluconazole & $0.5-8$ & 4 & 8 & 2.3123 \\
Itraconazole & $0.0313-3$ & 0.125 & 0.5 & 0.2816 \\
Total HIV- and HIV + & & & & \\
Amphotericin B & $0.0625-1$ & 0.5 & 1 & 0.4173 \\
Fluconazole & $0.125-8$ & 2 & 4 & 1.4385 \\
Itraconazole & $0.0313-3$ & 0.125 & 0.25 & 0.1321 \\
\hline
\end{tabular}

MIC50 $=$ Minimum Inhibitory Concentration required to inhibit the growth of 50\% of organisms, MIC90 $=$ Minimum Inhibitory Concentration required to inhibit the growth of $90 \%$ of organisms, GM= Geometric mean MIC 
The HIV-infected patients were younger compared with HIV-uninfected patients (mean age 30.2 years against 49.3 years respectively) and had CD4 counts $<200 / \mathrm{mm}^{3}$. The median CD4count among the HIV-infected patients was $32.5 / \mathrm{mm}^{3}$ (range: $8-109 / \mathrm{mm}^{3}$ ) and their median viral load was $6.18 \log$ copies $/ \mathrm{mL}$.

Cryptococcus neoformans isolated showed a uniform antifungal susceptibility pattern to the three antifungal

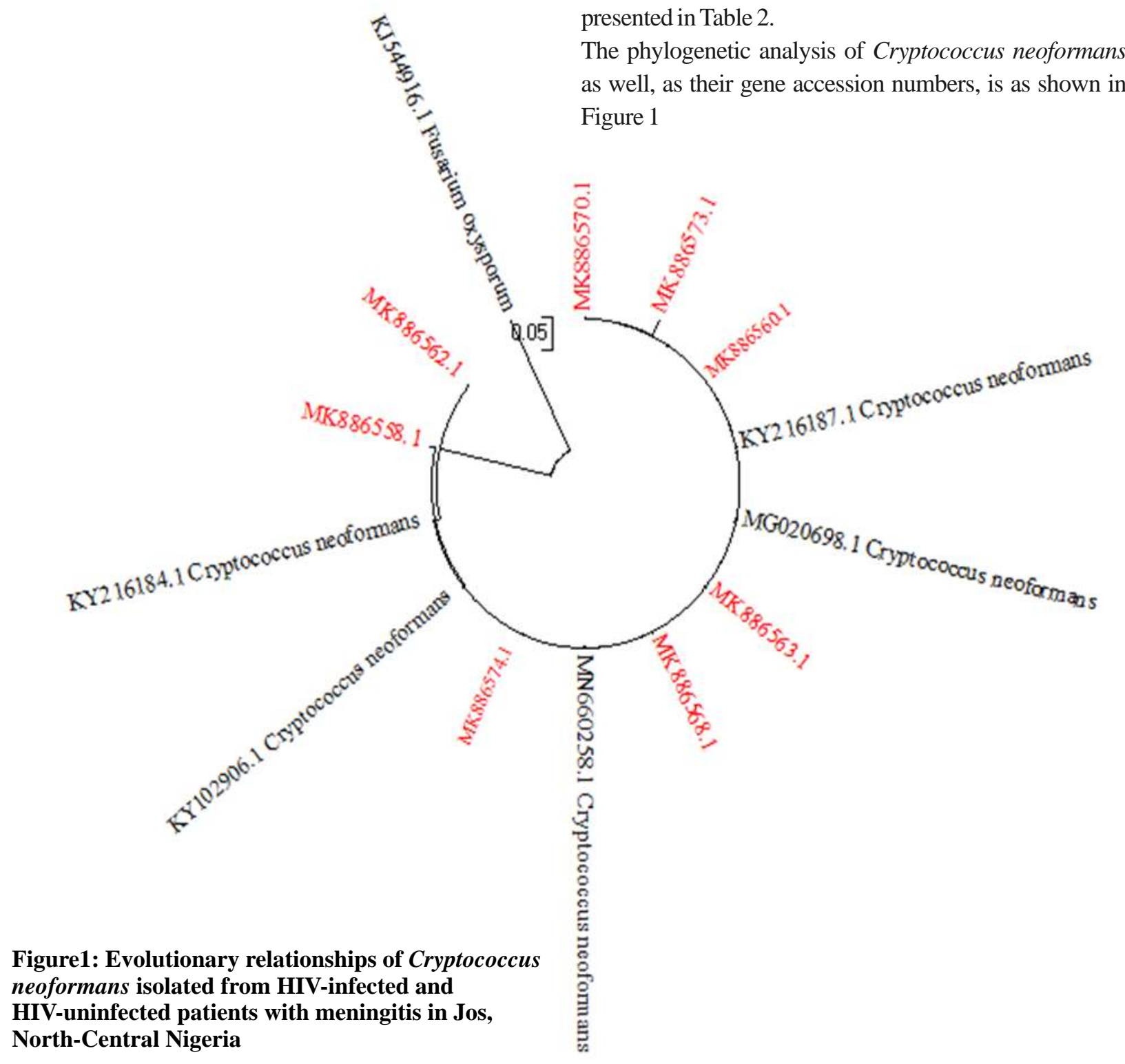

agents. The individual Minimum Inhibitory Concentration (MIC) ranges and MIC which inhibits $90 \%$ of the isolates $\left(\mathrm{MIC}_{90}\right)$ were $0.0313-3 \mu \mathrm{g} / \mathrm{ml}$ and $0.25 \mu \mathrm{g} / \mathrm{ml}$ for itraconazole, $0.065-1 \mu \mathrm{g} / \mathrm{ml}$ and $1 \mu \mathrm{g} / \mathrm{ml}$ for amphotericin B, $0.125-8 \mu \mathrm{g} / \mathrm{ml}$ and $4 \mu \mathrm{g} / \mathrm{ml}$ for fluconazole. The MIC ranges and $\mathrm{MIC}_{90}$ for itraconazole, amphotericin $\mathrm{B}$ and fluconazole were all within the reference range for the Cryptococcus neoformans isolated from HIV-infected and HIV-uninfected patients as presented in Table 2

The phylogenetic analysis of Cryptococcus neoformans as well, as their gene accession numbers, is as shown in Figure 1 


\section{DISCUSSION}

The prevalence of cryptococcal meningitis varies from one population to another. ${ }^{4,5,710,14,17}$ In our study, 31(16.8\%) of our patients with meningitis were all infected by Cryptococcus neoformans, whereas the prevalence among HIV-infected and HIV-uninfected patients were $13.4 \%$ and $18,8 \%$ respectively. Worldwide there is a variation in the distribution of Cryptococcus species causing meningitis ${ }^{1,29}$ but many of these studies have shown that Cryptococcus neoformans is the leading cause. ${ }^{7,14,18}$ It is now well established from a variety of studies that the prevalence of cryptococcal meningitis among HIV-infected patients is decreasing as compared with HIV-uninfected patients because the prevalence of HIV infection is equally decreasing and most of the HIVinfected patients are doing well on antiretroviral drugs. ${ }^{4,919}$ In keeping with several other studies, ${ }^{19,20}$ HIVinfected patients were significantly younger than HIVuninfected patients in our study. In both groups, males presented more with cryptococcal meningitis. Several other studies ${ }^{2,19,21}$ reported that HIV-infected patients with cryptococcal meningitis were mostly young males with Acquired Immunodeficiency Syndrome (AIDS), which may reflect a difference in the exposure of the patients rather than a difference in host susceptibility.

Amphotericin B has remained the mainstay of treatment for Cryptococcus neoformans for many years in both HIV-infected and HIV-uninfected patients. ${ }^{9,12,22}$ It targets the ergosterol in the plasma membrane of the fungus to form a channel that ultimately results in the disruption of the proton gradient. There are no defined breakpoints by the Clinical and Laboratory Standard Institute (CLSI) for amphotericin B and C. neoformans, it has been suggested that a Minimum Inhibitory Concentration (MIC) value of $2 \mu \mathrm{g} / \mathrm{ml}$ is the resistance threshold for amphotericin B and the susceptibility pattern of $C$. neoformans strain is predictable, with MICs ranging from $0.12-0.5 \mu \mathrm{g} / \mathrm{ml}^{23}$ Although our study did not identify any isolates with amphotericin B resistance, our result describes elevated average MICs to amphotericin B, which was rarely observed in other studies. ${ }^{6,11}$ It was reported that treatment with amphotericin B may induce the development of clinical and invitro amphotericin B resistance. The MIC values for fluconazole and itraconazole for both groups observed in this study were similar to those reported in some studies ${ }^{6,11,12,23}$ while a few others studies showed higher MIC values and increasing rates of resistance. ${ }^{12}$

Several phylogenetic studies evaluating the phylogeny of Cryptococcus species used single genomic loci such as (SSu), nuclear ribosomal large subunit (nucLSU), $5.8 \mathrm{~S}$ or ITS region, ${ }^{6,11,14}$ but our study utilized the ITS region which can also differentiate between the different species of cryptococcus. For accurate identification and therapeutic purposes, DNA-based techniques ${ }^{12}$ have been used to differentiate Cryptococcus species. Worthy of note is the fact that all of the ITS sequences used for the analysis in the study were generated from the PCR products. Regular epidemiological surveys that determine the genetic relationship among species of Cryptococcus and susceptibility patterns enables the identification of resistant strains. Our study also reinforces the utility of routine antifungal susceptibility studies for evaluating the patterns among Cryptococcus neoformans and its application for future drug therapeutic studies.

\section{CONCLUSION}

Cryptococcal meningitis is a treatable cause of death in both HIV-infected and HIV uninfected patients but it is often undiagnosed. A high index of suspicion in patients with known HIV-diagnosis, that are young and with features of meningitis should alert the possibility of cryptococcal meningitis and trigger aggressive diagnosis workups to rule out the disease and to consider empiric antifungal therapy.

\section{Acknowledgement}

Research reported in this abstract was supported by the Fogarty International Center and National Institute of 
Mental Health, of the National Institutes of Health under Award Number D43TW010543. The content is solely the responsibility of the authors and does not necessarily represent the official views of the National Institutes of Health.

\section{REFERENCES}

1. Rajasingham R, Smith RM, Park BJ, Jarvis JN, Govender NP, Chiller TM, et al. Global burden of disease of HIV-associated cryptococcal meningitis: an updated analysis. Lancet Infect Dis. 2017 Aug;17(8):873-81.

2. Mbuagbaw J, Jingi AM, Noubiap JJN, Kaze AD, Nansseu JRN, Bigna JJR, et al. Patterns and trends in mortality among HIV-infected and HIVuninfected patients in a major Internal Medicine Unit in Yaounde, Cameroon: a retrospective cohort study. JRSM open. 2016 Sep;7(9):2054270416654859.

3. Day JN, Qihui S, Thanh LT, Trieu PH, Van AD, Thu $\mathrm{NH}$, et al. Comparative genomics of Cryptococcus neoformans var. grubii associated with meningitis in HIV infected and uninfected patients in Vietnam. PLoS Negl Trop Dis. 2017 Jun;11(6):e0005628.

4. Lawrence DS, Boyer-Chammard T, Jarvis JN. Emerging concepts in HIV-associated cryptococcal meningitis. Curr Opin Infect Dis. 2019 Feb;32(1):16-23.

5. Fang W, Fa Z, Liao W. Epidemiology of Cryptococcus and cryptococcosis in China. Fungal Genet Biol. 2015 May;78:7-15.

6. Gago S, Serrano C, Alastruey-Izquierdo A, Cuesta I, Martin-Mazuelos E, Aller AI, et al. Molecular identification, antifungal resistance and virulence of Cryptococcus neoformans and Cryptococcus deneoformans isolated in Seville, Spain. Mycoses. 2017 Jan;60(1):40-50.

7. Cogliati M, Prigitano A, Esposto MC, Romano L, Grancini A, Zani A, et al. Epidemiological trends of cryptococcosis in Italy: Molecular typing and susceptibility pattern of Cryptococcus neoformans isolates collected during a 20-year period. Med Mycol. 2018 Nov;56(8):963-71.

8. Oladele RO, Bongomin F, Gago S, Denning DW. HIV-Associated Cryptococcal Disease in ResourceLimited Settings: A Case for "Prevention Is Better Than Cure"? J fungi (Basel, Switzerland). 2017 Dec;3(4).

9. Munivenkataswamy R, Gopi A, Usman SM, Jagadeesh. Human immunodeficiency virusassociated cryptococcal meningitis at a tertiary care centre: diagnostic tools and antifungal susceptibility testing. J Clin Diagn Res. 2013 Aug;7(8):1623-5.

10. Abhilash KPP, Mitra S, Arul JJJ, Raj PM, Balaji V, Kannangai R, et al. Changing paradigm of cryptococcal meningitis: an eight-year experience from a tertiary hospital in South India. Indian J Med Microbiol. 2015;33(1):25-9.

11. Hong N, Chen M, Xu N, Al-Hatmi AMS, Zhang C, Pan WH, et al. Genotypic diversity and antifungal susceptibility of Cryptococcus neoformans isolates from paediatric patients in China. Mycoses. 2019 Feb;62(2):171-80.

12. Herkert PF, Meis JF, Lucca de Oliveira Salvador G, Rodrigues Gomes R, Aparecida Vicente V, Dominguez Muro M, et al. Molecular characterization and antifungal susceptibility testing of Cryptococcus neoformans sensu stricto from southern Brazil. J Med Microbiol. 2018 Apr;67(4):560-9.

13. Siqueira LPM, Gimenes VMF, de Freitas RS, Melhem M de SC, Bonfietti LX, da Silva ARJ, et $a l$. Evaluation of Vitek MS for Differentiation of Cryptococcus neoformans and Cryptococcus gattii Genotypes. J Clin Microbiol. 2019 Jan;57(1).

14. Vanhove M, Beale MA, Rhodes J, Chanda D, Lakhi S, Kwenda G, et al. Genomic epidemiology of Cryptococcus yeasts identifies adaptation to environmental niches underpinning infection across an African HIV/AIDS cohort. Mol Ecol. 2017 
Apr;26(7):1991-2005.

15. Yoshida R, Nei M. Efficiencies of the NJp, Maximum Likelihood, and Bayesian Methods of Phylogenetic Construction for Compositional and Noncompositional Genes. Mol Biol Evol. 2016 Jun;33(6):1618-24.

16. Tamura K, Nei M, Kumar S. Prospects for inferring very large phylogenies by using the neighbor-joining method. Proc Natl Acad Sci U S A [Internet]. 2004 Jul 27;101(30):11030 LP 11035. Available from: http://www.pnas.org/content/101/30/11030.abstract

17. Chen J, Varma A, Diaz MR, Litvintseva AP, Wollenberg KK, Kwon-Chung KJ. Cryptococcus neoformans strains and infection in apparently immunocompetent patients, China. Emerg Infect Dis. 2008 May;14(5):755-62.

18. Hagen F, Khayhan K, Theelen B, Kolecka A, Polacheck I, Sionov E, et al. Recognition of seven species in the Cryptococcus gattii/Cryptococcus neoformans species complex. Fungal Genet Biol. 2015 May;78:16-48.

19. Tenforde MW, Wake R, Leeme T, Jarvis JN. HIVAssociated Cryptococcal Meningitis: Bridging the Gap Between Developed and Resource-Limited Settings. Curr Clin Microbiol Reports. 2016;3(2):92-102.

20. Chen J, Zhang R, Shen Y, Liu L, Qi T, Wang Z, et al. Serum cryptococcal antigen titre as a diagnostic tool and a predictor of mortality in HIV-infected patients with cryptococcal meningitis. HIV Med. 2019 Jan;20(1):69-73.

21. Yang R, Zhang H, Xiong Y, Gui X, Zhang Y, Deng $\mathrm{L}$, et al. Molecular diagnosis of central nervous system opportunistic infections and mortality in HIV - infected adults in Central China. AIDS Res Ther. 2017;1-7.

22. Rhodes J, Beale MA, Vanhove M, Jarvis JN, Kannambath S, Simpson JA, et al. A Population Genomics Approach to Assessing the Genetic Basis of Within-Host Microevolution Underlying
Recurrent Cryptococcal Meningitis Infection. G3 (Bethesda). 2017 Apr;7(4):1165-76.

23. Bejar V, Tello M, Garcia R, Guevara JM, Gonzales $\mathrm{S}$, Vergaray $\mathrm{G}$, et al. Molecular characterization and antifungal susceptibility of Cryptococcus neoformans strains collected from a single institution in Lima, Peru. Rev Iberoam Micol. 2015;32(2):88-92. 